\title{
Inducing formation of L10-phase in concave-cube FePt nanoparticles by annealing under high magnetic field
}

\section{Chun Wu}

Liaoning Technical University

\section{Weibin Zhuang}

Liaoning Technical University

Zhiyuan Niu

Liaoning Technical University

Dong Zhao

Northeastern University

Wenli Pei ( $\sim$ peiwl@atm.neu.edu.cn )

Northeastern University https://orcid.org/0000-0003-2525-152X

\section{Kai Wang}

Northeastern University

Qiang Wang

Northeastern University

\section{Nano Express}

Keywords: L10-FePt, anisotropy, high magnetic field, annealing, magnetic property

Posted Date: March 17th, 2020

DOI: https://doi.org/10.21203/rs.3.rs-17253/v1

License: (c) (i) This work is licensed under a Creative Commons Attribution 4.0 International License. Read Full License 


\section{Abstract}

Concave-cube FePt nanoparticles (NPs) with anisotropy of shape and element were annealed under high magnetic field (HMF). The high temperature sphered the FePt NPs, and Pt content and grain size of the NPs were decreased during annealing process. The HMF strength didn't affect the shape, size and composition of the FePt NPs, but induced the formation of L 10 -phase in the annealed NPs. The content and ordering degree of $L 10$-phase increased with enhancing the HMF strength, which leaded to the increasing of coercivity in the annealed FePt NPs. This work suggests that application of the HMF annealing is an effective strategy to tune the microstructure and property of anisotropic FePt NPs.

\section{Introduction}

For the wet-chemical synthesized Pt-based binary or multicomponent alloy nanoparticles (NPs), the growth of anisotropic shapes always leads to an anisotropic distribution of the elements [1, 2]. In the Pttransition binary alloy NPs, the Pt atom enriches at the corner (along $<111>$ direction), and the transition metal atoms prefer to deposit on the surfaces (along $<100>$ direction) [1]. The shape anisotropic NPs are well known to be sphered and transformed to shape isotropy during annealing process, however, what happens to the element distribution anisotropy? A type of convenient synthesized concave-cube FePt NP is a suitable model to solve this puzzle [3, 4]. The shape anisotropy of concave-cube FePt NPs is originated from the over-growth of truncated-cube or cube seeds along $<111>$ direction, and the Pt contents is richer at corner-site, which leads to the element distribution anisotropy [5-7]. Otherwise, the as-synthesized FePt NPs always shows a disorder fcc structure, the annealing is required to form an orderly L $1_{0}$-phase with better magnetic and electrocatalytic performances [8-11]. The typical anisotropy of shape and element, and the meaningful disorder-order transition of concave-cube fcc-FePt NPs offer us a good option to study the microstructure and property evolution of anisotropic NPs during annealing.

Recently, high-magnetic-field-assisted (HMF-assisted) heat treatment method attracts more and more attentions with the developing of superconducting technology. The HMF presents special effects on tailoring the morphology of films [12,13], regulating the growth rates of crystals [14-17], and operating the thermal dynamic and kinetic of solid-state phase transformation [18-20]. For the FePt alloys, the $\mathrm{HMF}$ annealing has been reported to induce strain in $\mathrm{Fe}_{3} \mathrm{Pt}$ alloy [21], promote disorder-order transformation of FePt films [22, 23], and alignment of FePt spherical NPs [24, 25]. Fewer researchers address the influences of HMF annealing on the NPs with the shape and element distribution anisotropy, and it still is an open issue in this field.

In this paper, the wet-chemical synthesized concave-cube FePt NPs with shape and element distribution anisotropy have been annealed under the HMF, and effects of the HMF on shape, size, composition, crystal structure and magnetic properties of the FePt NPs have been investigated. It is expected to reveal the effects of the HMF annealing on anisotropic structure.

\section{Experimental Method}


The concave-cube FePt NPs were synthesized by a wet-chemical method. Typically, $0.25 \mathrm{mmol} \mathrm{Pt}(\mathrm{acac})_{2}$, $25 \mathrm{ml}$ benzyl ether and $0.25 \mathrm{~g} \mathrm{1,2-hexadecanediol} \mathrm{were} \mathrm{mixed,} \mathrm{and} \mathrm{heated} \mathrm{to} 105^{\circ} \mathrm{C}$ to remove moisture. Surfactants $3 \mathrm{ml} \mathrm{OA}, 3 \mathrm{ml} \mathrm{OAm}$ and precursor $\mathrm{Fe}(\mathrm{CO})_{5}(0.5 \mathrm{mmol})$ were injected into the hot solution, and then heated to $220^{\circ} \mathrm{C}$ at the rate of $3{ }^{\circ} \mathrm{C} / \mathrm{min}$. After refluxing for $60 \mathrm{~min}$, the mixture was naturally cooled to room temperature. Finally, the NPs were washed repeatedly with ethanol, hexane, and dispersed in hexane with concentration about $5 \mathrm{mg} / \mathrm{ml}$. The wet-chemical process was repeated several times to obtain enough FePt NPs.

The annealing process was as follows. About $30 \mathrm{ml}$ of FePt-hexane mixture, $50 \mathrm{ml}$ hexane and $60 \mathrm{~g} \mathrm{NaCl}$ (ball milling, smaller than $22 \mathrm{um}$ ) were mixed and dried slowly by magnetic stirring at $80^{\circ} \mathrm{C}$. Then, the FePt NPs-NaCl mixture was divided into three parts with equal mass to insurance the uniformity of NPs contents during annealing. The as-synthesized FePt NPs were annealed at $700^{\circ} \mathrm{C}$ for $1 \mathrm{~h}$ in vacuum, which was placed inside a super-conducting $12 \mathrm{~T}$ magnet. After annealed, the NPs were collected through centrifuge with water and alcohol, and stored in alcohol at $-20^{\circ} \mathrm{C}$.

The shape and size of FePt NPs were characterized by Transmission Electron Microscopy (TEM, JEM$2100 \mathrm{~F}$ ) at an accelerating voltage of $200 \mathrm{kV}$. Size distributions were collected through counting at least 100 particles in TEM images by using Win Roof software. The composition of the FePt NPs was analyzed by Field Emission Scanning Electronic Microscopy (FE-SEM, SUPRA 35) associated Energy Dispersive Spectroscopy. The magnetic properties were measured by Vibrating Sample Magnetometer at room temperature on a Micro Sense EZ9 magnetometer.

\section{Results And Discussion}

Figure 1 (a) shows the TEM image of as-synthesized FePt NPs, the NPs are typical concave-cube shape with uniform size. TEM images of the samples after annealing under 0, 6 and $12 \mathrm{~T} \mathrm{HMF}$ are showed in Fig. 1 (b) (d), respectively. From the TEM images, it can be found that the shape-anisotropic concavecube NPs transform to sphere after annealing, the size uniformity is poor and some NPs had grown abnormally in the annealed samples. Although the $\mathrm{NaCl}$ medium is employed to avoid aggregation of the NPs during annealing, the random abnormal growth of the NPs is still unavoidable [11,26]. As shown in Fig. $1\left(\mathrm{e}_{1}\right)$ and $\left(\mathrm{e}_{2}\right)$, the lattice fringes of as-synthesized and 0 T-annealed FePt nanoparticles display interplanar spacing of 0.20 and $0.27 \mathrm{~nm}$ in the particle, which match well respectively with those of the (111) and the (110) planes of the $A 1$ and $L 1_{2}$ phases. The SAED patterns of as-synthesized and $12 \mathrm{~T}$ annealed NPs are showed in Fig. $1\left(f_{1}\right)$ and $\left(f_{2}\right)$, respectively. After annealed at $12 \mathrm{~T}$, the diffraction rings of (001), (110) and (002) faces are collected, which indicates that the L $1_{0}$-FePt NPs are generated at $12 \mathrm{~T}$.

The grain sizes of the NPs are collected from TEM images, the size distributions are showed in Fig. 2(a). The distributions of the NPs fit well with Gauss Function, average size of the as-synthesized NPs is $11.55 \mathrm{~nm}$, and most of sizes are between 9 and $14 \mathrm{~nm}$. After annealed, the size distributions are moved to 
left-side, most sizes are between 5 and $11 \mathrm{~nm}$, and the grain size of the FePt NPs decreases to about $8.51 \mathrm{~nm}$. The average sizes of the NPs annealed under 0, 6 and $12 \mathrm{~T} \mathrm{HMF}$ are consistent within the error range, which means the HMF strength shows no effect on sizes of the annealed NPs.

The EDS patterns of the NPs are showed in Fig. 2 (b). It should be noticed that the relative intensity of Pt $L_{a}$ peak decreases after annealing, and the Fe content in the NPs is increased from $51.6 \%$ to about $72.1 \%$, which means the Pt atoms in FePt NPs are decreased after annealing. Generally, the composition of the NPs is consistent before and after annealing. In this research, the as-synthesized concave-cube FePt NPs are shape and element anisotropic, the NPs will become spherical and the Pt-rich corners will fall off from the FePt concave-cubic NPs during annealing. Due to the higher Pt content and smaller size of the corners, it is hard to be collected through centrifugation, and lead to the decreasing of Pt content in the NPs.

Fig. 3 shows the XRD patterns of as-synthesized and post-annealed FePt NPs. The as-synthesized FePt NPs is $A 1-F e P t$ phase with a disorder $f c c$ structure [9]. After annealing without of the HMF, the diffraction peaks of (001), (110) and (201) faces are detected, considering the composition of the NPs closes to $\mathrm{Fe}_{70} \mathrm{Pt}_{30}$, the peaks can be indexed as $L 1_{2}-\mathrm{Fe}_{3} \mathrm{Pt}$ phase. To diffraction patterns of samples applied the 6 and 12 T HMF during annealing, a remarkable change can be found that the (002) peak of A1-FePt divides into two peaks, which indicates that the $L 1_{0}$-FePt phase forms at the 6 and 12 T HMF. The separation of (200) and (002) peaks is the most important characteristic of $L 1_{0}-\mathrm{FePt}$, the distance between those two peaks relates to the ordering degree of $L 1_{0}-\mathrm{FePt}$, which suggest that the ordering degree of $L 1_{0}$-FePt is obviously enhanced at the $12 \mathrm{~T} \operatorname{HMF}[26,27]$.

The ordering degree $s$ of the NPs calculates by $s=0.85 \times\left[\mathrm{I}_{(001)} / \mathrm{I}_{(002)}\right]^{0.5}$, where $\mathrm{I}_{(001)}$ and $\mathrm{I}_{(002)}$ are the intensity of (001) and (002) peaks, respectively. The s increases from 0.46 to 0.65 when increasing the HMF from 6 to $12 \mathrm{~T}$, also indicating that the ordering degree can be enhanced by increasing HMF strength. Both the diffraction peaks of $\mathrm{L} 1_{2}-\mathrm{Fe}_{3} \mathrm{Pt}$ and $\mathrm{L} 1_{0}-\mathrm{FePt}$ can be found in the HMF-annealed samples, the content of $\mathrm{L} 1_{0}$-FePt phase in the samples annealed at the 6 and $12 \mathrm{~T}$ has been roughly calculated and it increases from 16.8-25.1\%, which means the increasing of the HMF strength also increases the content of the $\mathrm{L} 1_{0}-\mathrm{FePt} N P s$. The crystal structure of the concave-cube A1-FePt NPs transforms to $L 1_{2}-\mathrm{Fe}_{3} \mathrm{Pt}$ after annealed, application of the HMF will induce formation of $L 1_{0}-\mathrm{FePt}$, the ordering degree and the content of $L 1_{0}-\mathrm{FePt}$ increases with the enhancing of HMF strength.

The room-temperature hysteresis loops of the as-synthesized and the post-annealed FePt NPs are showed in Fig. 4. The nonzero coercivity is detected in the post-annealed FePt NPs, it means the assynthesized superparamagnetic FePt NPs transform to ferromagnetic after annealing. While application of the 0, 6 and 12 T HMF during annealing, the coercivity of the FePt NPs increases from 266 to 363 and 
489 Oe, respectively. The coercivity of the annealed FePt NPs relates to the grain size and effective anisotropy [23]. Although the HMF shows no effects on grain sizes of NPs, the HMF induces formation and ordering degree enhancement of $L 1_{0}$-FePt NPs increase the effective anisotropy and coercivity of FePt NPs. The coercivity of the sample annealed at $12 \mathrm{~T}$ is not so high, because the larger component deviation (far from 1:1) will lead to the less content and lower ordering degree of $L 1_{0}$-phase in the annealed FePt NPs [23, 26, 27].

The effects of HMF annealing on concave-cube FePt NPs are summarized in Fig. 5. The as-synthesized concave-cube FePt are both shape and element anisotropic, the Pt atoms rich in the corner-site. After annealing, the shape anisotropic FePt NPs become spherical, the Pt-rich corner is fell off from the concave-cube FePt NPs. As the mass of the Pt-rich corner is too small to be centrifugal collected, the Pt content and grain size of the annealed FePt NPs is decreased. The Fe/Pt atoms ratio of FePt NPs closes to 3:1 after annealing, which leads to the formation of orderly $L 1_{2}-\mathrm{Fe}_{3} \mathrm{Pt}$ phase. This result also indicates that the temperature of spheroidization of concave-cube FePt NPs is lower than that of disorder-order transformation temperature, the Pt-rich corner are fell off from the NPs firstly, and then the residual Fe and Pt atoms are diffused to form the ordered phase.

The strength of the HMF cannot affect the shape, size and composition evolutions of FePt NPs during annealing, but induces the formation of $\mathrm{L} 1_{0}-\mathrm{FePt} N P s$. In the equilibrium phase diagram of FePt alloys, the maximum Fe content in $\mathrm{L} 1_{0}$-phase is about $70 \%[27,28]$. The Fe content in annealed NPs is about $72.1 \%$, closes to the critical value, which suggests the possibility of formation $L 1_{0}-F e P t ~ N P s$. As the temperature of spheroidization is lower, the Pt-rich corner are fell off still. However, the HMF prefers the formation of $L 1_{0}$-phase, because the magnetocrystalline anisotropy energy of $L 1_{0}$-phase is higher than that of $L 1_{2}$-phase. The nucleation of $L 1_{0}$-phase and ordering diffusion of $\mathrm{Fe} / \mathrm{Pt}$ atoms will be enhanced by the HMF induced magnetization energy [19-23]. Therefore, the content and ordering degree of $L 1_{0}{ }^{-}$ FePt in the annealed NPs is increased by increasing HMF strength. However, as the Fe/Pt atoms ratio of the annealed FePt NPs is far from 1/1, only a very small amount of $L 1_{0}$-FePt NPs with lower orderly degree can be generated.

\section{Conclusions}

The wet-chemical synthesized concave-cube FePt NPs with shape and element distribution anisotropy were annealed under 0, 6 and 12 T HMF. The concave-cube FePt NPs became spherical, the Pt-rich cornersite fell off from the NPs, and the grain sizes were decreased during the annealing. The ferromagnetic $\mathrm{L}_{2}{ }_{2}-\mathrm{Fe}_{3} \mathrm{Pt}$ NPs were formed after annealed at $0 \mathrm{~T}$. The HMF didn't influence on the shape, size and composition of the NPs. The orderly L1 $1_{0}-$ FePt NPs were generated in the NPs annealed at 6 and $12 \mathrm{~T}$ 
HMF. The ordering degree, content, and the coercivity of $L 1_{0}-F e P t$ NPs increased with enhancing the HMF strength, which suggests that the nucleation of $L 1_{0}$-phase and ordering diffusion of $\mathrm{Fe} / \mathrm{Pt}$ atoms were promoted and tuned by the HMF. This research provided a promising method to control the microstructure and property for anisotropic NPs.

\section{Declarations}

Competing Interests

The authors declare that they have no competing interests.

Authors' Contributions

CW, and WZ performed the experiments, analyzed the data, and drafted the manuscript. ZN and DZ participated in the preparation of the FePt NPs. WP and KW helped to finish the sample characterization. The whole project was under the direction of QW. All authors read and approved the final manuscript.

Acknowledgments

This research was financially supported by the National Natural Science Foundation of China (Grant Nos. 51871045, 51690161), the Liaoning Innovative Research Team in University (Grant No. LT2017011), the Basic Research Program of the Key Laboratory of Liaoning Province (No. LZ2015035), the Fundamental Research Funds for the Central Universities (Grant Nos. N160907001, N180912004), and the Doctoral Start-up Foundation of Liaoning Province (Grant No. 2019-BS-116).

\section{References}

1.

Gan L, Cui C, Heggen M et al (2014) Element-specific anisotropic growth of shaped platinum alloy nanocrystals. Science 346:1502-1506

2.

Prabhudev S, Bugnet M, Zhu GZ et al (2015) Surface segregation of Fe in Pt-Fe alloy nanoparticles: its precedence and effect on the ordered-phase evolution during thermal annealing. ChemCatChem 7:36553664

3.

Wu J, Zhu J, Zhou M et al (2012) FePt concave nanocubes with enhanced methanol oxidation activity. CrystEngComm 14:572-7575

4.

Wang DY, Chou HL, Cheng CC et al (2015) FePt nanodendrites with high-index facets as active electrocatalysts for oxygen reduction reaction. Nano Energy 11:631-639

5 .

Chou SW, Zhu CL, Neeleshwar S et al (2009) Controlled growth and magnetic property of FePt nanostructure: cuboctahedron, octapod, truncated cube, and cube. Chem Mater 21:4955-4961 6. 
Wu C, Pei W, Wang X et al (2016) High magnetic field-induced synthesis of one-dimensional FePt nanomaterials. RSC Adv 6:84684-84688

7.

Duan X, Wu C, Wang X et al (2019) Evolutions of microstructure and magnetic property of wet-chemical synthesized FePt nanoparticles assisted by high magnetic field. J Alloys Compd 797:1372-1377

8.

Sun SH, Murray CB, Weller D et al (2000) Monodisperse FePt nanoparticles and ferromagnetic FePt nanocrystal superlattices. Science 287:1989-1992

9.

Zeng H, Li J, Liu JP et al (2002) Exchange-coupled nanocomposite magnets by nanoparticle selfassembly. Nature 420:395-398

10.

Li Q, Wu L, Wu G et al (2015) New approach to fully ordered fct-FePt nanoparticles for much enhanced electrocatalysis in acid. Nano Lett 15:2468-2473

11.

Pei W, Zhao D, Wu C et al (2019) Facile liquid-assisted one-step sintering synthesis of super fine $L 1_{0}$-FePt nanoparticles. RSC Adv 9:36034-36039

12.

Li D, Levesque A, Franczak A et al (2013) Evolution of morphology in electrodeposited nanocrystalline Co-Ni films by in-situ high magnetic field application. Talanta 110:66-70

13.

Li G, Du J, Wang H et al (2014) High magnetic field induced pillar growth and subsequent magnetic properties of the thermal evaporated Co thin films. Mater Lett 133:53-56

14.

Wu C, Wang X, Pei W et al (2019) Tailoring the shape and size of wet-chemical synthesized FePt nanoparticles by controlling nucleation and growth with a high magnetic field. Nanoscale 11:1502315028

15.

Wang M, Chen Q (2010) Experimental and theoretical investigations on the magnetic-field-induced variation of surface energy of $\mathrm{Co}_{3} \mathrm{O}_{4}$ crystal faces. Chem Eur J 16:12088-12090 16.

Daltin AL, Chopart JP (2010) Microcrystals electrodeposited in a high magnetic field. Cryst Growth Des $10: 2267-2271$

17.

Xu Y, Ren Z, Ren W et al (2008) Magnetic-field-assisted solvothermal growth of single-crystalline bismuth nanowires. Nanotechnology 19:115602

18.

Wu C, Wang K, Li D et al (2016) Tuning microstructure and magnetic properties of electrodeposited CoNiP films by high magnetic field annealing. J Magn Magn Mater 416:61-65

19. 
Ma Y, Xiao L, Yan L (2006) Application of high magnetic fields in advanced materials processing. Chinese Sci Bull 51:2944-2950

20.

Hu L, Zhang R, Chen Q (2014) Synthesis and assembly of nanomaterials under magnetic fields. Nanoscale 6:14064-14105

21.

Fukuda T, Yamamoto M, Yamaguchi T et al (2014) Magnetocrystalline anisotropy and magnetic fieldinduced strain of three martensites in $\mathrm{Fe}_{3} \mathrm{Pt}$ ferromagnetic shape memory alloys. Acta Mater 62:182-187 22.

Liu L, Lv H, Sheng W et al (2012) Orientation control in L1 0 FePt films by using magnetic field annealing around Curie temperature. Appl Surf Sci 258:5770-5773

23.

Wang HY, Ma XK, He YJ et al (2004) Enhancement in ordering of FePt films by magnetic field annealing. Appl Phys Lett 85:2304-2306

24.

Kura H, Sato T, Takahashi M et al (2008) Effect of coalescence on ordering and c-axis orientation of FePt, FePtAu, and FePtCu nanoparticles heat-treated in magnetic field. Jpn J Appl Phys 47:3466-3470 25.

Tamada Y, Yamamoto S, Nasu S et al (2008) Structural and magnetic properties of L1 $1_{0}-\mathrm{FePt}$ nanoparticles aligned by external magnetic field. Phys Rev B 78:214428

26.

He J, Bian B, Zheng Q et al (2016) Direct chemical synthesis of well dispersed L $1_{0}$-FePt nanoparticles with tunable size and coercivity. Green Chem 18:417-422

27.

Liu Y, Jiang Y, Zhang X et al (2014) Structural and magnetic properties of the ordered $\mathrm{FePt}_{3}, \mathrm{FePt}$ and $\mathrm{Fe}_{3} \mathrm{Pt}$ nanoparticles. J Solid State Chem 209:69-73

28.

Gutfleisch O, Lyubina J, Müller KH et al (2005) FePt hard magnets. Adv Eng Mater 7:208-212

\section{Figures}




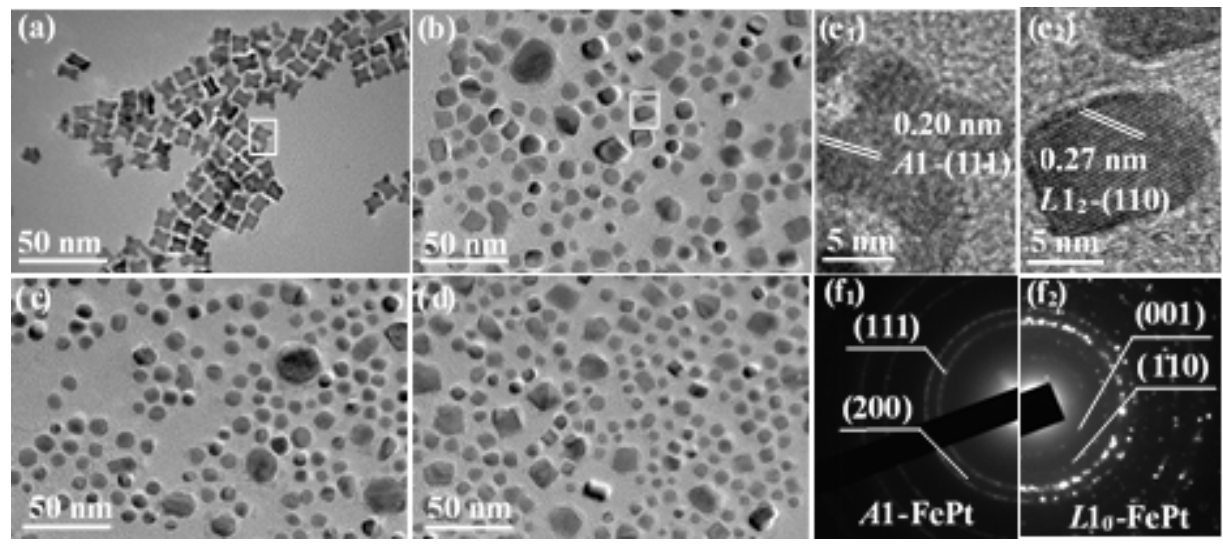

Figure 1

TEM images of (a) as-synthesized and post-annealed FePt nanoparticles at magnetic field of (b) 0 T, (c) 6 T, (d) 12 T. HRTEM images of FePt nanoparticles, (e1) from the white box in (a); (e2) from the white box in (b). SAED patterns of (f1) as-synthesized and (f2) 12 T-annealed nanoparticles.
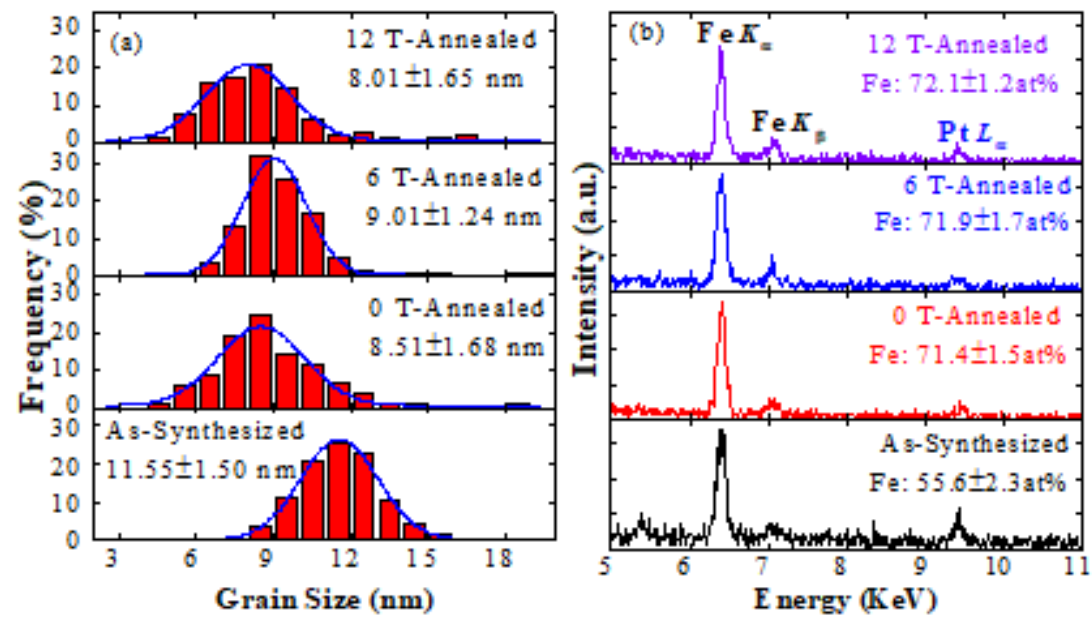

\section{Figure 2}

(a) Grain sizes distributions and (b) EDS patterns of as-synthesized and post-annealed FePt nanoparticles.

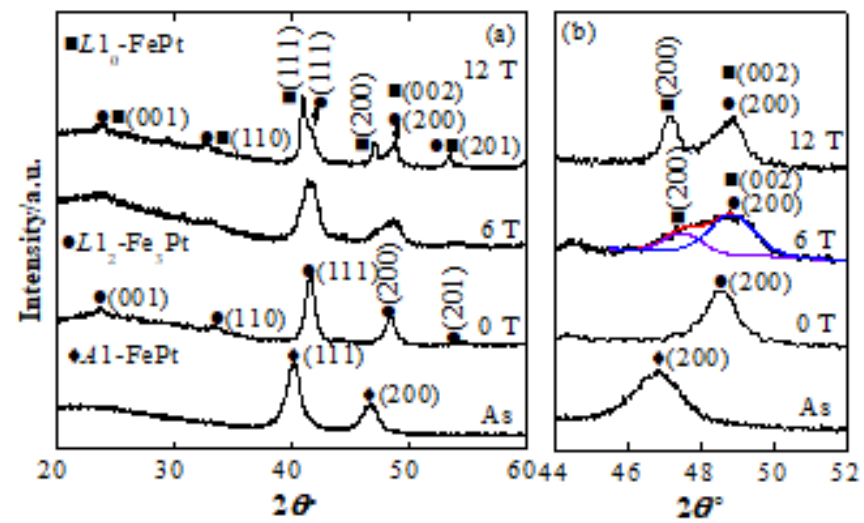

Figure 3 
(a) XRD patterns of as-synthesized and post-annealed FePt nanoparticles. (b) Right side shows the enlarge patterns from $44^{\circ}$ to $52^{\circ}$.

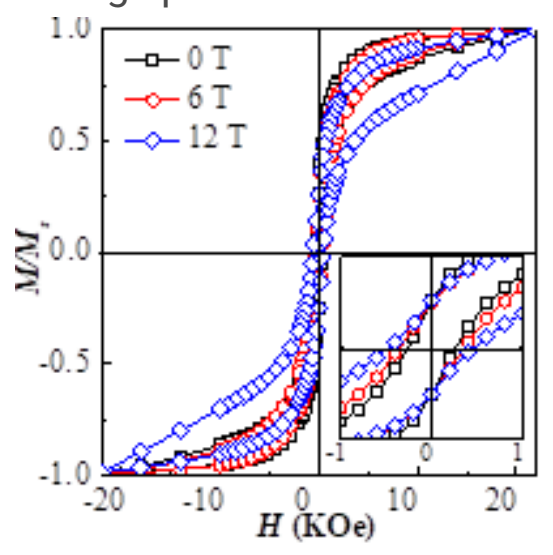

Figure 4

Hysteresis loops of as-synthesized and post-annealed FePt nanoparticles. Inset figure shows the enlarge loops from -1 to $1 \mathrm{kOe}$.

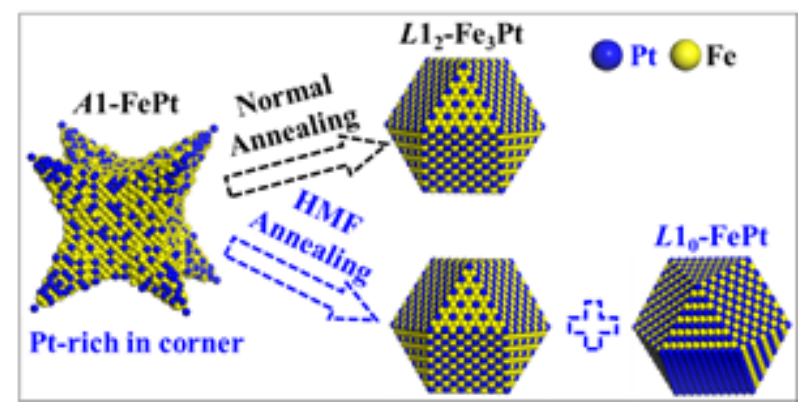

Figure 5

A schematic diagram illustrating the effects of HMF annealing on microstructure of the concave-cube FePt NP. 